
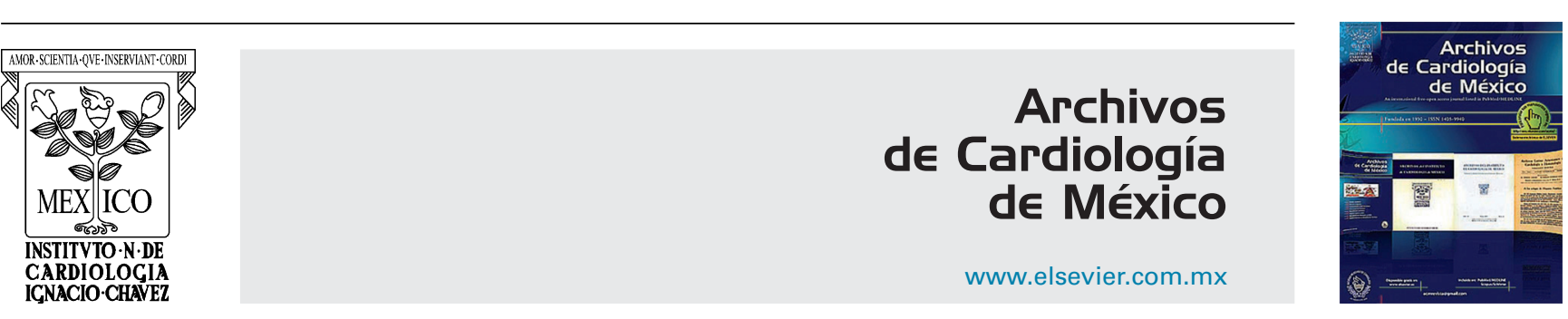

INVESTIGACIÓN CLÍNICA

\title{
Situación del intervalo QTc en el período neonatal en un hospital del occidente de México, estudio piloto
}

\section{Rocio Alejandra Peña-Juárez ${ }^{a, *}$, Adrián Garcia-Canales ${ }^{\mathrm{b}}$, Luis Martin Garrido-García ${ }^{\mathrm{c}}$ y Cesar Augusto Valerio-Carballoc}

a Cardiología Pediátrica, Hospital General de Occidente, Zapopan, Jalisco, México

b Terapia Intensiva Pediátrica, Hospital Regional Valentín Gómez Farías, Zapopan, Jalisco, México

c Cardiología Pediátrica, Instituto Nacional de Pediatría, Coyoacán, Ciudad de México, México

Recibido el 4 de abril de 2017; aceptado el 30 de septiembre de 2017

\section{PALABRAS CLAVE Intervalo QTC; Recién nacido; Arritmias; Electrocardiograma; México}

\begin{abstract}
Resumen
Introducción: La prolongación del intervalo QT se asocia con arritmias ventriculares y muerte súbita.

Objetivo: Determinar el valor del intervalo QTc en recién nacidos sanos y evaluar su comportamiento durante los primeros meses de vida.

Métodos: Recién nacidos sanos a término de marzo-noviembre 2016 en el Hospital General de Occidente del Estado de Jalisco, México. Se les practicó un electrocardiograma de 12 derivaciones a una velocidad de $25 \mathrm{~mm} / \mathrm{s}$ durante las primeras $48 \mathrm{~h}$. Se midió el intervalo QT en DII y se calculó el QTc mediante la fórmula de Bazett. Los pacientes a los que se detectó prolongación del QTc en sus primeras $48 \mathrm{~h}$ se valoraron al mes con ECG y ecocardiograma. Si persistieron con QTc alargado se revaloraron a los 6 meses con ECG, Holter de $24 \mathrm{~h}$ y estudio electrocardiográfico a padres y hermanos. Los que persistieron con QTc alargado se revaloraron a los 9 meses con ECG.

Resultados: Se incluyeron 548 pacientes. La media del intervalo QTc al nacimiento fue de $459 \mathrm{~ms}$; durante este período 33 pacientes presentaron un QTc mayor de $470 \mathrm{~ms}$, los cuales fueron evaluados al mes mediante un nuevo estudio electrocardiográfico, obteniendo una media del intervalo QTC de $446 \mathrm{~ms}$. A los 6 meses se evaluaron 16 pacientes, con una media del intervalo QTC de $434 \mathrm{~ms}$. A los 9 meses se evaluaron 6 pacientes, con una media del intervalo QTC de $438 \mathrm{~ms}$, y solo 4 pacientes persistieron con QTc prolongado.

Conclusiones: El intervalo QTc en nuestros pacientes es más prolongado en comparación con otras poblaciones y muestra una normalización paulatina.

(c) 2017 Instituto Nacional de Cardiología Ignacio Chávez. Publicado por Masson Doyma México S.A. Este es un artículo Open Access bajo la licencia CC BY-NC-ND (http://creativecommons. org/licenses/by-nc-nd/4.0/).
\end{abstract}

\footnotetext{
* Autor para correspondencia. Avenida Zoquipan 1050, Colonia Seattle, CP 45650, Zapopan, Jalisco, México. Teléfono: +01333334534721. Correo electrónico: alepejz@gmail.com (R.A. Peña-Juárez).
} 


\section{KEYWORDS}

QTc interval; Newborn; Arrhythmias; Electrocardiogram; Mexico
QTC interval in the neonatal period in a Mexican population. A pilot study

\section{Abstract}

Introduction: QT interval prolongation is associated with ventricular arrhythmias and sudden death syndrome.

Objective: To determine the value of QTC interval in healthy newborns in a general hospital in Jalisco, Mexico, and their outcome during their first months of life.

Methods: The study included healthy newborns from March to November 2016, in the Hospital General of Occidente in Jalisco, Mexico. A 12-lead electrocardiogram was performed at a speed of $25 \mathrm{~mm} / \mathrm{s}$ during the first $48 \mathrm{~h}$ of life. The QT interval was measured in lead DII, and the QTc interval was calculated using the Bazett formula. Patients detected with QTc prolongation were assessed monthly with an ECG and echocardiogram. If they persisted with prolonged QTC interval, they were re-evaluated at 6 months with an ECG, $24 \mathrm{~h}$ Holter, and electrocardiography study on parents and siblings. Those who persisted with prolonged QTc interval were evaluated with an ECG at 9 months.

Results: The study included 548 patients. The mean QTc interval at birth was 459 ms; during this period 33 patients has a QTc greated that 470 ms; which were evaluated monthly with a new electrocardiographic study, obtaining a mean QTc interal of 446 ms. At 6 months 16 patients were evaluated, with a mean QTc interval of $434 \mathrm{~ms}$. At 9 months, 6 patients were found to have a mean QTC interval of $438 \mathrm{~ms}$, and only 4 patients persisted with a prolonged QTC interval. Conclusions: The QTC interval in our population is prolonged compared to other populations and with a gradual return to normal.

(c) 2017 Instituto Nacional de Cardiología Ignacio Chávez. Published by Masson Doyma México S.A. This is an open access article under the CC BY-NC-ND license (http://creativecommons. org/licenses/by-nc-nd/4.0/).

\section{Introducción}

La muerte súbita en la etapa pediátrica ocurre aproximadamente de 0.8 a 6.2 por cada 100,000 niños. El diagnóstico de alteraciones cardiacas que pudieran ocasionarla ha sido objeto de estudio en los últimos años. La realización de un electrocardiograma (ECG) en el período neonatal como herramienta diagnóstica generalizada aún está en discusión, ya que la asociación de intervalo QT prolongado y muerte súbita es difícil de determinar debido a la presencia de formas ocultas o latentes ${ }^{1}$. Además, se han observado grados variables de presentación, que se hacen evidentes con uso de fármacos u otras situaciones clínicas ${ }^{2}$. Estudios recientes han reportado que los valores del intervalo QT son distintos dependiendo del origen étnico y esto es debido a diferencias genéticas ${ }^{3}$. Se han realizado varios estudios donde evaluaron la utilidad del ECG como tamizaje neonatal. En el metaanálisis realizado por Rodday et al. se evaluaron 6,954 trabajos en los cuales se estudiaron 100,000 pacientes asintomáticos buscando enfermedades como miocardiopatía hipertrófica, síndrome QT largo y síndrome de Wolff-Parkinson-White. Se encontró que el ECG y el ecocardiograma tienen una alta sensibilidad y un elevado valor predictivo para detectar enfermedades cardiacas, pero con la presencia de falsos positivos variables ${ }^{1}$. Vetter reportó que la realización de un ECG en la etapa neonatal puede ayudar a determinar causas de muerte súbita y prevenir efectos adversos ${ }^{4}$. Sin embargo, estas recomendaciones deben considerarse con base en las situaciones de los distintos centros. Además, existe la duda del momento de la realización del estudio electrocardiográfico, ya que se ha observado que la duración del intervalo
QT no tiene una medición uniforme. Schwartz et al. reportaron que el intervalo QT presenta un alargamiento fisiológico y temporal durante el primer mes de vida ${ }^{5}$. En México, no existen estudios sobre el valor del intervalo QT en niños normales, así como tampoco sobre el momento de la toma del ECG para la detección de anomalías del ritmo.

\section{Métodos}

Este es un estudio prospectivo, longitudinal, observacional, el cual se realizó de marzo a noviembre del 2016 en un hospital de segundo nivel de atención del occidente de México. Se incluyeron recién nacidos sanos, a término (37 a 41.6 semanas de gestación), con peso adecuado para la edad gestacional (mayor de 2,500 g), hijos de madres sanas, obtenidos por vía vaginal, con calificación de Apgar al minuto mayor de 8. Se descartaron aquellos pacientes cuyas madres habían recibido algún tipo de anestésico o medicamento que prolongue el intervalo QT y también pacientes con prueba de oximetría anormal. Se les tomó un ECG de superficie de 12 derivaciones en las primeras $48 \mathrm{~h}$ de vida. Se evaluó la frecuencia cardiaca con método de estimación, se midió el intervalo QT en DIl y se calculó el QT corregido (QTC) mediante la fórmula de Bazett por 2 cardiólogos pediatras de manera independiente utilizando cálculo manual y la aplicación Qx Calculate ${ }^{T M}$.

Se consideró alargamiento de QTC un valor mayor de 470 ms durante los primeros 6 meses y, posterior a ello, de $440 \mathrm{~ms}$. Los pacientes a los que se detectó alargamiento de QTc en las primeras $48 \mathrm{~h}$ se valoraron al mes con ECG y ecocardiograma. Si estos pacientes se encontraban con 
QTc mayor de $440 \mathrm{~ms}$, se revaloraron nuevamente a los 6 meses con ECG, Holter de $24 \mathrm{~h}$ y estudio electrocardiográfico a padres y hermanos. Los que persistieron con intervalo QTC mayor de $440 \mathrm{~ms}$ a los 6 meses se revaloraron solo con ECG a los 9 meses.

Este estudio fue aprobado por el Comité de Investigación y Ética del hospital.

\section{Resultados}

Durante el período de estudio se evaluaron 548 pacientes. La edad de gestación promedio fue de $38.6 \pm 1.4$ semanas, y el peso promedio fue de $3,220 \pm 422 \mathrm{~g}$. De los pacientes estudiados, 287 fueron masculinos (52.4\%). Se descartaron antecedentes de muerte súbita en todos los pacientes (tabla 1). Al nacimiento, la frecuencia cardiaca promedio fue de $140 \pm 16 \mathrm{lpm}$, con un intervalo QT medido de $316.7 \pm 39.7 \mathrm{~ms}$ y un intervalo QTc de $459 \pm 51 \mathrm{~ms}$. Se encontró un intervalo QTc prolongado en 33 pacientes (6\%). No se encontraron diferencias significativas en cuanto al género. En los pacientes masculinos la media del intervalo QTC fue de $456 \mathrm{~ms}$ en comparación con el género femenino, en que fue de $455 \mathrm{~ms}(p<0.709)$. Al mes de edad, se evaluaron 33 pacientes, con una frecuencia cardiaca promedio de $138 \pm 16 \mathrm{lpm}$, un intervalo QT medido de $309 \pm 29 \mathrm{~ms}$ y un intervalo QTc de $446 \pm 34 \mathrm{~ms}$. El ecocardiograma demostró un corazón estructuralmente sano en 23 pacientes (69.6\%). En 4 pacientes se diagnosticó una comunicación interauricular (12.1\%), en 5 pacientes, un conducto arterioso (15.1\%) y en un paciente, una comunicación interventricular (3\%) (tabla 2).

De los 33 pacientes evaluados al mes de edad, 16 (48.4\%) persistieron con un QTC prolongado. A 16 pacientes se les tomó un nuevo ECG a los 6 meses de edad, y se encontró que tuvieron una frecuencia cardiaca promedio de $143 \pm 17$ lpm, un intervalo QT medido de $295 \pm 28 \mathrm{~ms}$ y un intervalo QTc de $434 \pm 32 \mathrm{~ms}$. En el estudio Holter de $24 \mathrm{~h}$ no se encontró ninguna alteración importante del ritmo. La frecuencia cardiaca máxima fue de $189 \pm 13 \mathrm{lpm}$, la frecuencia cardiaca mínima, de $102 \pm 9$ lpm, el intervalo QT máximo fue de $449 \pm 17 \mathrm{~ms}$ y el intervalo QT mínimo, de $386 \pm 41 \mathrm{~ms}$. Las medias de los resultados fueron: QT máximo $449 \pm 17 \mathrm{~ms}$, QT mínimo $386 \pm 41 \mathrm{~m}$, frecuencia

Tabla 1 Datos demográficos $(n=548)$ de los pacientes al nacimiento

\begin{tabular}{ll}
\hline Peso, $g$ & $3,220 \pm 422$ \\
Semanas de gestación & $38.6 \pm 1.4$ \\
Género, \% & Masculino 52.4 \\
& Femenino 47.6 \\
\hline
\end{tabular}

Tabla 2 Hallazgos ecocardiográficos en los pacientes con QT alargado

\begin{tabular}{ll}
\hline Hallazgo ecocardiográfico & Porcentaje \\
\hline Corazón sano & 69.6 \\
Conducto arterioso permeable & 15.1 \\
Comunicación interauricular & 12.1 \\
Comunicación interventricular & 3 \\
\hline
\end{tabular}

Tabla 3 Valores de electrocardiograma medidos durante los primeros 9 meses

\begin{tabular}{lllll}
\hline Valores & $48 \mathrm{~h}$ & Un mes & 6 meses & 9 meses \\
\hline Pacientes, $\mathrm{n}$ & 548 & 33 & 16 & 6 \\
$\begin{array}{l}\text { QT medido }(\mathrm{ms}), \\
\quad 316.7 \pm 39.7\end{array}$ & $309 \pm 29$ & $295 \pm 28$ & $289 \pm 17$ \\
$\quad \begin{array}{l}\text { media } \pm \mathrm{DE} \\
\text { QT corregido }(\mathrm{ms}),\end{array}$ & $459 \pm 51$ & $446 \pm 34$ & $434 \pm 32$ & $438 \pm 21$ \\
$\quad$ media $\pm \mathrm{DE}$ & & & & \\
$\begin{array}{l}\text { Frecuencia } \\
\quad \text { cardiaca }(\mathrm{lpm}),\end{array}$ & $140 \pm 16$ & $138 \pm 16$ & $143 \pm 17$ & $144 \pm 12$ \\
$\quad$ media $\pm \mathrm{DE}$ & & & & \\
\hline
\end{tabular}

cardiaca máxima $189 \pm 13 \mathrm{lpm}$, frecuencia cardiaca mínima $102 \pm 9$ lpm. No se reportó ningún trastorno del ritmo. El estudio electrocardiográfico de los padres y hermanos no reportó alteración alguna en el intervalo QTc.

A los 9 meses se valoraron 6 pacientes, los cuales presentaban un QTc mayor de $440 \mathrm{~ms}$. La frecuencia cardiaca promedio fue de $144 \pm 12 \mathrm{lpm}$, el intervalo QT medido, de $289 \pm 17 \mathrm{~ms}$, y el intervalo QTc, de $438 \pm 21 \mathrm{~ms}$ (tabla 3 ).

\section{Discusión}

El intervalo QT representa la repolarización ventricular, y su alargamiento puede condicionar arritmias ventriculares letales, pudiendo ser la primer manifestación la muerte súbita $^{6}$. Como se ha mencionado en la literatura, el intervalo QTc presenta una normalización paulatina, siendo más prolongado durante la etapa neonatal y asociándose a diferencias étnicas ${ }^{3,7}$. Nuestros resultados mostraron que la media del intervalo QTC durante la etapa neonatal fue de $459 \mathrm{~ms}$, que es mayor que lo reportado en la literatura.

La causa de la prolongación transitoria del intervalo QT durante la etapa neonatal no ha sido determinada. Las 2 teorías más importantes para explicar este fenómeno son:

1) Hipótesis del QT. Refiere que existe un desbalance en la inervación simpática, caracterizada por una dominancia de la inervación en el lado izquierdo que predispone a fibrilación ventricular y que se asocia con un alargamiento temporal del intervalo $\mathrm{QT}^{5}$.

2) Inestabilidad de la repolarización cardiaca. En un estudio experimental se observó que durante la maduración miocárdica existe inestabilidad de la repolarización durante el desarrollo posnatal, el cual pudiera ser el sustrato susceptible de arritmias fatales. Estos cambios dinámicos en la repolarización provocan una alternancia eléctrica, ocasionando cambios en la duración del potencial de la acción, siendo un sello distintivo de inestabilidad eléctrica, lo cual se ha correlacionado en el ECG con un mayor riesgo de arritmias y una menor supervivencia a largo plazo en adultos ${ }^{8}$.

También se ha observado una prolongación del intervalo QT en enfermedades donde existe un incremento de la presión del ventrículo derecho, como en el caso de pacientes operados de corrección de tetralogía de Fallot con insuficiencia pulmonar severa. En estos pacientes, se incrementa la mortalidad secundaria al desarrollo de arritmias ventriculares por un alargamiento del intervalo QTc ${ }^{9}$. Este mismo fenómeno se ha observado en pacientes con hipertensión pulmonar severa, como han reportado Rich et al., que encontraron una asociación entre la prolongación del QTC y 


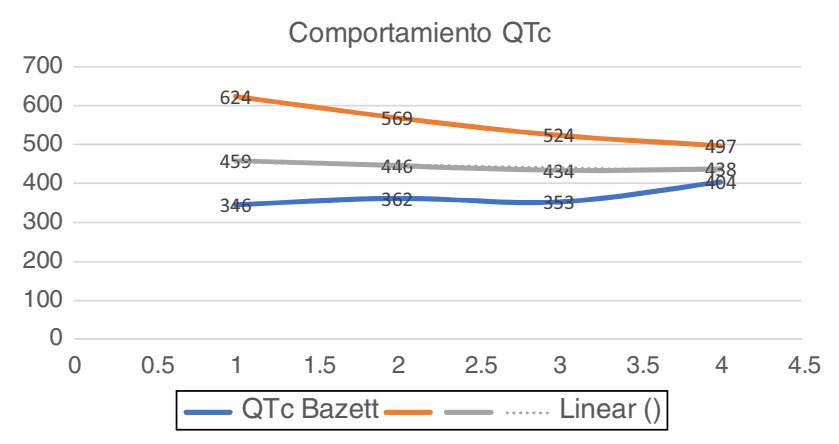

Figura 1 Se observa una disminución paulatina del QTC durante los primeros meses de vida.

una alteración en la función ventricular derecha ${ }^{10}$. También encontraron que los pacientes con QTc alargado correlacionaron directamente con un incremento en el volumen diastólico final del ventrículo derecho y la masa ventricular derecha. Además, el intervalo QTc fue inversamente proporcional a la fracción de eyección ventricular derecha.

El incremento de la presión ventricular derecha pudiera ser el causante del alargamiento transitorio del QTC en la etapa neonatal. En esta etapa el ventrículo derecho es el dominante por la presencia de resistencias pulmonares elevadas ${ }^{11}$, condicionando un incremento fisiológico de la presión ventricular derecha y, con ello, una ampliación en el período diastólico y en la duración de la fase 3 del potencial de acción, ocasionando un incremento de pospotenciales tardíos y, por lo tanto, un alargamiento del intervalo QT durante la etapa neonatal. Al disminuir de manera fisiológica las resistencias pulmonares, se disminuye la presión ventricular derecha y, por lo tanto, existe una «normalización» en el potencial de acción, lo cual explica la disminución en la duración del intervalo QT que observamos en nuestro estudio, que es de hasta 25 ms en los primeros 6 meses de edad (fig. 1). Esta disminución paulatina se ha reportado en otros estudios, como el de Schwartz et al., en el cual se observó una disminución del intervalo QTc en los primeros 6 meses. La mayoría de los pacientes con QTc prolongados al nacimiento presentaron normalización del QTc en el segundo mes de vida ${ }^{5}$.

Por otro lado, es posible que el intervalo QTc en las primeras $48 \mathrm{~h}$ de vida sea normalmente largo por alteraciones en la maduración del sistema nervioso autónomo y que se normalice en el primer mes. Se ha observado también que el rango del intervalo QTc puede cambiar con base en el origen étnico, lo que probablemente refleje diferencias genéticas sin que esto suponga una enfermedad ${ }^{3}$.

La prolongación del intervalo QT corregido es una entidad clínica asociada a muerte súbita, lo que ha motivado intensamente su estudio. En nuestro trabajo, se encontraron valores prolongados del intervalo QT; sin embargo, no se inició tratamiento médico debido a que ningún paciente presentó factores de riesgo para muerte súbita (tamiz auditivo normal y familiares sin antecedente de muerte súbita).

Actualmente, el síndrome de QT prolongado congénito se considera una canalopatía secundaria a múltiples mutaciones genéticas y con un riesgo elevado de muerte súbita ${ }^{6}$, por lo que ante el hallazgo de un intervalo QT prolongado en un ECG en el recién nacido está indicada la realización de un estudio genético que corrobore el diagnóstico de la enfermedad. Este estudio no pudo realizarse en nuestros pacientes, ya que no está disponible en nuestro medio.

El hallazgo de diferentes cardiopatías en nuestros pacientes no se consideró como causa de la prolongación del intervalo QT.

Un punto importante que tenemos que tomar en cuenta es decidir qué fórmula utilizar para la corrección del intervalo QT con base en la frecuencia cardiaca. Sabemos que para pacientes con frecuencias cardiacas mayores de $110 \mathrm{lpm}$, la Food and Drug Administration recomienda utilizar la fórmula de Frederica. Sin embargo, la fórmula de Bazett es la más utilizada en la práctica clínica y es empleada en todas las definiciones del síndrome de QTC prolongado. Existen múltiples estudios donde se valora la corrección del QT medido con base en la frecuencia cardiaca; uno de los más importantes es el de Phan et al. ${ }^{12}$, en donde estudiaron 702 pacientes y compararon 4 escalas para valorar el QTc con base en la frecuencia cardiaca, encontrando que el QTc varía al utilizar la fórmula de Bazett en comparación con la de Frederica. Sin embargo, se concluye que la fórmula de Bazett no solo provee una mejor corrección del QTc, sino que además mejora el rango para identificar el síndrome de QT prolongado, el cual se consideró en 460 ms. Además, en el Consenso Europeo, que es la única guía publicada para la valoración del ECG neonatal, se utilizó la fórmula de Bazett para la valoración del intervalo QTc ${ }^{13}$. Tomando como base estos estudios, decidimos la valoración del QTC por la fórmula de Bazett.

En la mayoría de las definiciones de intervalo QT prolongado se toma como referencia un intervalo mayor de $440 \mathrm{~ms}$; de hecho, en muchos estudios neonatales se recomienda iniciar el estudio del QT prolongado a partir de dicha cifra ${ }^{13}$. Sin embargo, estos valores están descritos en pacientes adultos y no toman en cuenta la mayor duración del intervalo QT en el recién nacido. Para nuestro estudio decidimos tomar un intervalo de $470 \mathrm{~ms}$ como definición de QT prolongado con base en el reporte de Martí-Almor et al. ${ }^{3}$, en donde valoraron el intervalo QTC en recién nacidos de distintos grupos étnicos y encontraron que el valor de $470 \mathrm{~ms}$ fue el más adecuado para definir normalidad y alteración del QTC.

El primer ECG realizado en nuestros pacientes fue en el período neonatal inmediato (en las primeras $48 \mathrm{~h}$ de vida), donde se sabe que el intervalo QT tiene una duración mayor y después presenta una disminución en la duración. De hecho, se han reportado estudios en donde se refiere que la duración máxima del intervalo QTc es entre las 6 y las 11 semanas, siendo este un punto importante de debate en cuanto al momento de realización del $\mathrm{ECG}^{7}$.

\section{Conclusiones}

Los valores del intervalo QTC en nuestro estudio fueron más altos en comparación con los de estudios previos; sin embargo, presentaron un descenso paulatino a valores normales en las evaluaciones posteriores.

No obstante, ningún paciente presentó complicaciones secundarias a la prolongación del intervalo QT. En aquellos en los que persistió la prolongación del intervalo QTC es necesaria la realización de un estudio genético para corroborar la existencia de alguna mutación y un seguimiento a largo plazo. 
Las principales limitaciones del estudio fueron el número reducido de pacientes y el no poder realizar la prueba genética a aquellos con intervalos QTc prolongados.

\section{Financiación}

No se recibió patrocinio de ningún tipo para llevar a cabo este artículo.

\section{Conflicto de intereses}

Los autores declaran no tener ningún conflicto de intereses.

\section{Bibliografía}

1. Rodday AM, Triedman JK, Alexander ME, et al. Electrocardiogram screening for disorders that cause sudden cardiac death in asymptomatic children: A meta-analysis. Pediatrics. 2012;129:e999-1010.

2. Zayas-Molina R. Actualización sobre el síndrome de QT largo congénito. Rev Cubana Invest Biomed. 2012;31:129-44.

3. Martí-Almor J, Berrueco R, García-Algar O, et al. Intervalo QT en recién nacidos de diferente origen étnico: utilidad del cribado con ECG neonatal. Rev Esp Cardiol. 2008;61:980-2.

4. Vetter VL. Should electrocardiographic (ECG) screening of all infants, children, and teenagers be performed? Circulation. 2014;130:688-97.
5. Schwartz PJ, Montemerlo M, Facchini M, et al. The QT interval throughout the first 6 months of life: A prospective study. Circulation. 1982;66:496-501.

6. Medeiros-Domingo A, Iturralde-Torres P, Ackerman MJ. Clinica y genética en el síndrome de QT largo. Rev Esp Cardiol. 2007;60:739-52.

7. Yoshinaga M, Kato Y, Nomura Y, et al. The QT intervals in infancy and time for infantile ECG screening for long QT syndrome. J Arrhythm. 2011;27:193-201.

8. Idriss SF, Bell JA. Cardiac repolarization inestability during normal postnatal development. J Electrocardiol. 2008;41: 474-9.

9. Sabate-Rotes A, Connolly HM, Warnes CA, et al. Ventricular arrhytmia risk stratification in patients with tetralogy of Fallot at the time of pulmonary valve replacement. Circ Arrhythm Electrophysiol. 2015;8:110-6.

10. Rich JD, Thenappan T, Freed B, et al. QTC prolongation is associated with impaired right ventricular function and predicts mortality in pulmonary hypertension. Int J Cardiol. 2013;167:669-76.

11. Brockmeier K, Nazal R, Sreeram N. The electrocardiogram of the neonate and infant. J Electrocardiol. 2016;49: 814-6.

12. Phan DQ, Silka MJ, Lan YT, et al. Comparison of formulas for calculation of the corrected QT interval in infants and young children. J Pediatr. 2015;166:960-4.

13. Schwartz P, Garson A Jr, Paul T, et al. Guidelines for the interpretation of the neonatal electrocardiogram. Eur Heart J. 2002;23:1329-44. 